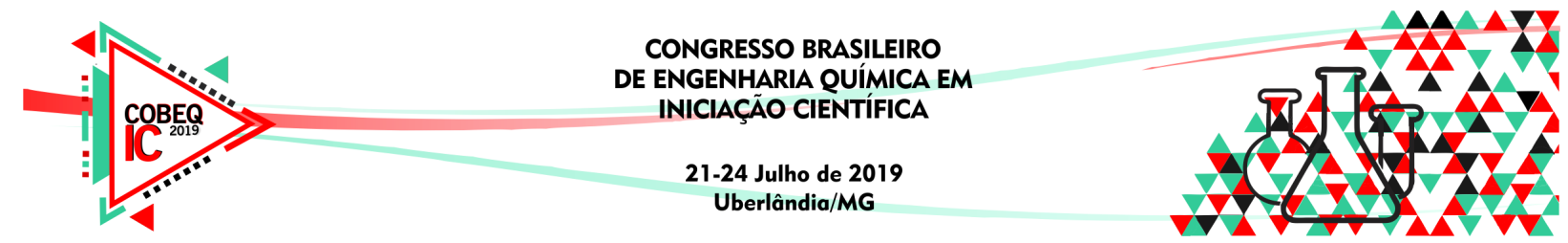

\title{
SOLUÇÃO NUMÉRICA PARA O PROBLEMA DE FILTRAÇÃO TANGENCIAL COM MALHAS NÃO- UNIFORMES
}

\author{
D. E. N. LIMA ${ }^{1}$ e J. M. SILVA ${ }^{1}$ \\ ${ }^{1}$ Universidade Federal de Alfenas, Instituto de Ciência e Tecnologia \\ E-mail para contato: douglasjales33@gmail.com
}

\begin{abstract}
RESUMO - Nesse trabalho, objetiva-se avaliar o desempenho de malha estruturada e não-uniforme em relação a uma malha uniforme para os resultados numéricos da concentração de partículas sólidas em escoamentos em tubos permeáveis, o qual está relacionado ao processo de filtração tangencial. Para isso, a equação de convecção-difusão, relacionada a equação de transporte de massa, será discretizada utilizando-se a técnica de diferenças finitas.
\end{abstract}

\section{INTRODUÇÃO}

Durante o processo de filtração tangencial, partículas dentro do fluxo de alimentação são convectivamente dirigidas para a superfície da membrana onde elas acumulam, enquanto que o movimento difusivo de partículas causa um transporte, dessas partículas, oposto ao transporte convectivo do escoamento de filtrado (permeado) (MIGNARD e GLASS, 2001; RIPPERGER e ALTMANN, 2002) (ver Figura 1).

Figura 1: Direção de escoamento dos processos da filtração tangencial (RIPPERGER e ALTMANN, 2002).

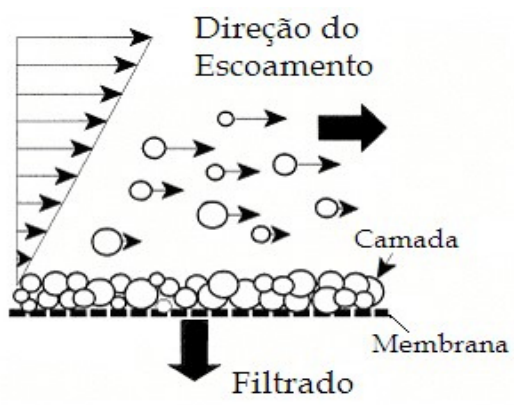

O acúmulo de partículas próximo à superfície da membrana é conhecido como concentração de polarização, o qual é um fenômeno que causa uma complicação adicional ao processo de filtração, pois provoca o aumento na resistência hidráulica para o fluxo de permeado e, consequentemente, o declínio do fluxo com o tempo. Por isso, um melhor conhecimento da formação da camada e da deposição de partículas na superfície permeável poderia resultar em um uso econômico da filtração tangencial em muitas aplicações técnicas (ZEMAN e ZYDNEY, 1996; RIPPERGER e ALTMANN, 2002). Assim, uma variedade de 


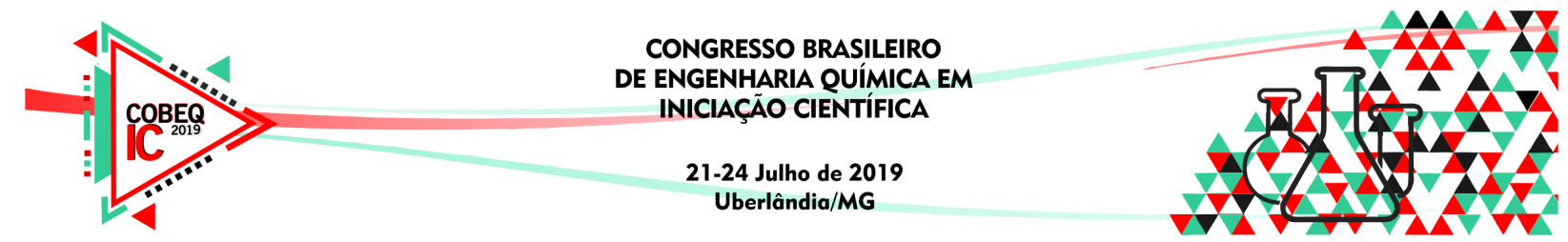

trabalhos dedicados à modelagem numérica para analisar a concentração de polarização durante o processo de filtração tangencial é encontrada na literatura (DAMAK, K. et al., 2004; VENEZUELA et al., 2009; SILVA et al., 2011).

Como a região próxima a parede permeável é onde os valores da concentração são mais altos, então, o uso de uma malha computacional não-uniforme com um refinamento mais denso na região próxima à parede permeável do tubo permitirá uma melhor análise da camada de polarização. Portanto, dentro desse cenário, esse trabalho de pesquisa visa avaliar os resultados numéricos quando se utiliza uma malha computacional estruturada e não-uniforme para definir, durante um escoamento em tubo permeável, aplicado ao processo de filtração tangencial, a concentração de partículas próxima à superfície permeável.

\section{FORMULAÇÃO MATEMÁTICA}

Nesta investigação será considerado um escoamento incompressível, isotérmico e completamente desenvolvido em tubo cilíndrico com paredes permeáveis. A formulação matemática adimensional é determinada pela equação diferencial de convecção-difusão, a qual representa a equação de conservação das espécies químicas

$$
u \frac{\partial c}{\partial z}=\frac{1}{\operatorname{Re} S c}\left(\frac{1}{r} \frac{\partial c}{\partial r}+\frac{\partial^{2} c}{\partial r^{2}}\right),
$$

em que $u$ é a velocidade axial, $u$ é a velocidade axial, $c$ é a concentração de soluto, Re é o número de Reynolds ( $\operatorname{Re}=\rho u_{0} d / \mu ; \rho$ é a densidade do fluido, $u_{0}$ é a velocidade axial de entrada, $d$ é o diâmetro da tubulação e $\mu$ é a viscosidade dinâmica do fluido), $S c$ é o número de Schmidt ( $S c=\mu / \rho D$; D a difusividade de massa) e $r$ e $z$ são as coordenadas radial e axial, respectivamente.

\subsection{CONDIÇÕES DE FRONTEIRA}

As condições de fronteira para velocidade e concentração de soluto na forma adimensional são definidas por (DAMAK et al., 2004; GERALDES et al., 2001; ZEMAN e ZYDNEY, 1996)

( i ) entrada

$$
z=0,0 \leq r \leq 1, u=1, c=1
$$

( ii ) eixo de simetria

$$
r=0,0 \leq z \leq L / R, \frac{\partial u}{\partial r}=0, \frac{\partial c}{\partial r}=0
$$

em que $L$ e $R$ são o comprimento e o raio do tubo, respectivamente; 
( iii ) superfície permeável

$$
r=1,0 \leq z \leq L / R, u=0, c_{w} v_{w}=\left.\frac{1}{\operatorname{Re} S c} \frac{\partial c}{\partial r}\right|_{r=R},
$$

em que $c_{w}$ é a concentração de soluto na superfície permeável e $v_{w}$ é a velocidade de permeação, a qual é constante nesse trabalho;

(iv ) saída

$$
z=\mathrm{L}, 0 \leq r \leq 1, \frac{\partial u}{\partial z}=0, \frac{\partial c}{\partial z}=0
$$

\subsection{MÉTODO NUMÉRICO}

A Equação (1) juntamente com as condições de fronteira, Equações (2) - (5), foram discretizadas pela técnica de diferenças finitas em malha computacional co-localizada estruturada e não-uniforme, em que todos os elementos (células) são retangulares de largura $\Delta z$ e altura $\Delta r_{j}$ e as variáveis são definidas no mesmo ponto, como mostra a Figura 2.

Figura 2 - Malha estruturada e não-uniforme.

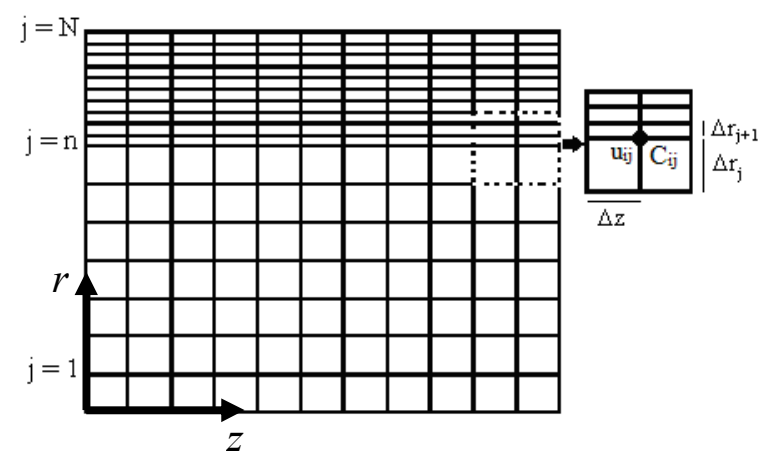

As derivadas dos termo $z$ onvectivos da Equação (1) foram aproximadas por diferenças atrasadas

$$
\left.u \frac{\partial c}{\partial z}\right|_{i, j}=u_{i, j}\left(\frac{c_{i, j}-c_{i-1, j}}{\Delta z}\right)
$$

e as derivadas do termo difusivo foram aproximadas considerando-se a malha computacional não-uniforme em relação a coordenada radial (FERZIGER e PERIC, 2002)

$$
\left.\frac{\partial c}{\partial r}\right|_{i, j} \approx \frac{\Delta r_{j} c_{i, j+1}-\Delta r_{j+1} c_{i, j-1}+\left(\Delta r_{j+1}-\Delta r_{j}\right) c_{i, j}}{2 \Delta r_{j+1} \Delta r_{j}},
$$




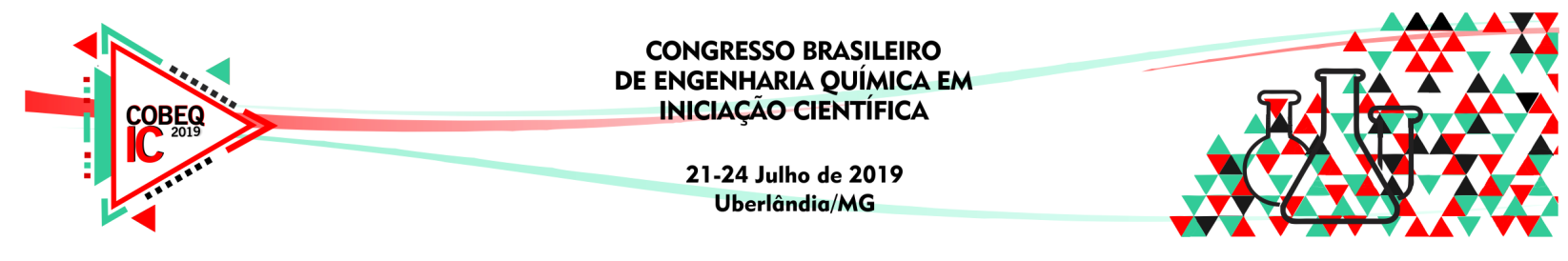

$$
\left.\frac{\partial^{2} c}{\partial r^{2}}\right|_{i, j} \approx \frac{2\left[\Delta r_{j} c_{i, j+1}+\Delta r_{j+1} c_{i, j-1}-\left(\Delta r_{j+1}+\Delta r_{j}\right) c_{i, j}\right]}{\left(\Delta r_{j}+\Delta r_{j+1}\right) \Delta r_{j+1} \Delta r_{j}}
$$

em que $\Delta r_{j}$ é o tamanho dos elementos da malha não-uniforme no eixo $r$.

A discretização da Equação (1) resultou em um sistema de equações lineares, o qual foi resolvido pelo método iterativo de Gauss-Seidel (FRANCO, 2007).

\section{RESULTADOS E DISCUSSÕES}

Após discretização e implementação das Equações (1) - (5), algumas simulações foram realizadas utilizando-se $R=0,015 \mathrm{~m}, L=0,0375 \mathrm{~m}, v_{w}=1,36 \times 10^{-6} \mathrm{~ms}^{-1}$ e malhas computacionais em que $70 \%$ da quantidade total de pontos do eixo radial foram distribuídos próximo à superfície permeável (representada por $30 \%$ do raio do tubo, $R$ ). Após várias simulações para diferentes malhas computacionais, identificou-se que não há mais alterações significativas nos perfis de concentração a partir da malha de $1500 \times 400$ pontos. Portanto, nas próximas simulações utiliza-se tal malha como padrão.

Na Figura 3, apresentam-se, a comparação entre o resultado numérico adimensional do perfil de concentração em função da coordenada axial com os resultados analítico-numérico obtido por Venezuela et al. (2009), para $R e=600$ e $S c=1429$. Observa-se que os resultados numéricos apresentam uma boa tendência de comportamento com os resultados da literatura, com erro relativo médio de 5,4\%. A discordância entre o resultado teórico e numérico pode ser atribuída ao fato do trabalho de Venezuela et al. (2009) possuir a parcela radial convectiva, $\partial c / \partial r$, na Equação (1). Observou-se também que a malha computacional não uniforme convergiu com 259 iterações, enquanto que a malha uniforme converge com 572 iterações, com o mesmo $\Delta r$ da região mais densa da malha não uniforme.

Figura 3 - Comparação do perfil de concentração próximo à superfície permeável em função da coordenada axial com os resultados obtidos da literatura.

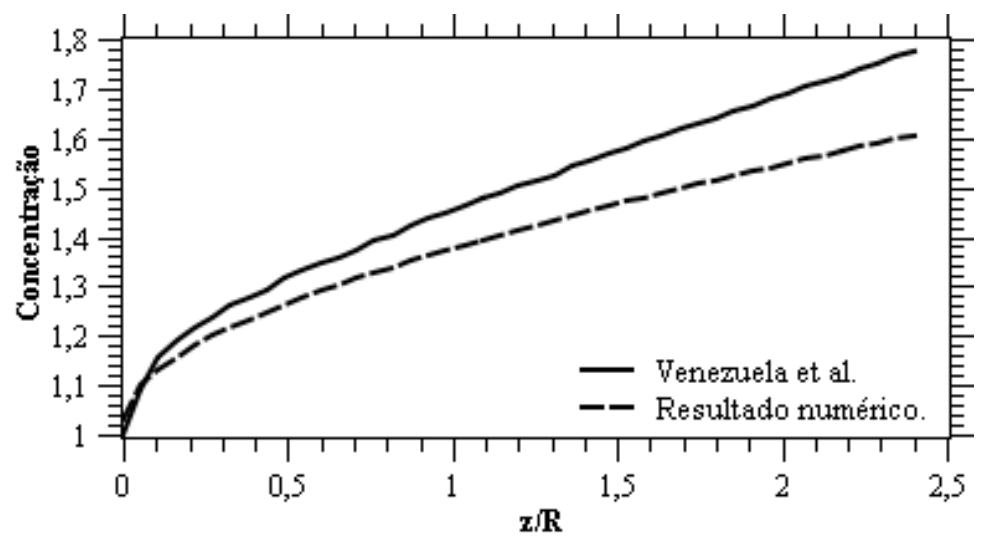

Nas Figuras 4 e 5 apresentam-se os resultados numéricos para a espessura da camada de polarização em função da coordenada axial para diferentes números de Schmidt e 


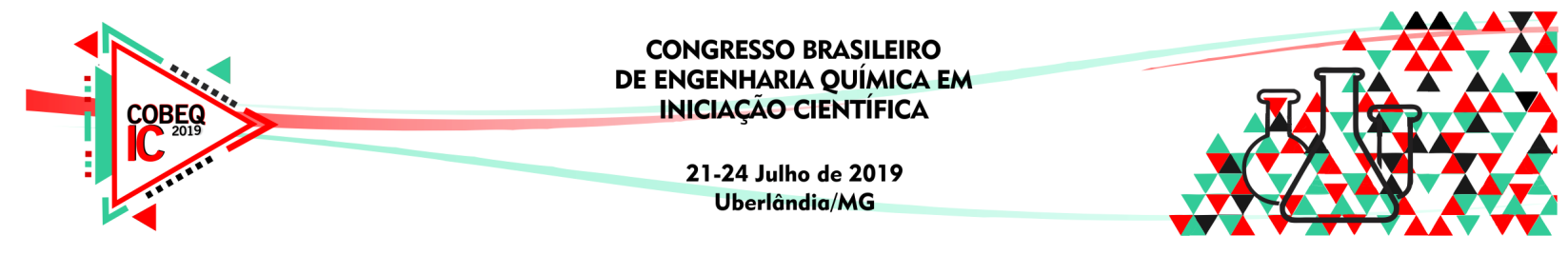

Reynolds, respectivamente. O cálculo da espessura foi definido como a distância da parede permeável ao local onde $\left(c-c_{0}\right) / c_{0} \leq 10^{-3}$ (DAMAK et al., 2004), em que $c_{0}$ é a concentração inicial.

Nota-se na Figura 4 que o aumento do número de Schmidt causa uma diminuição da camada de polarização. Isso se deve ao fato que, de acordo com a definição, o número de Schmidt é inversamente proporcional a difusividade, ou seja, quanto maior o número de Schmidt menor a difusividade de partículas.

Figura 4 - Espessura da camada de polarização em função da coordenada axial para diferentes números de Schmidt.

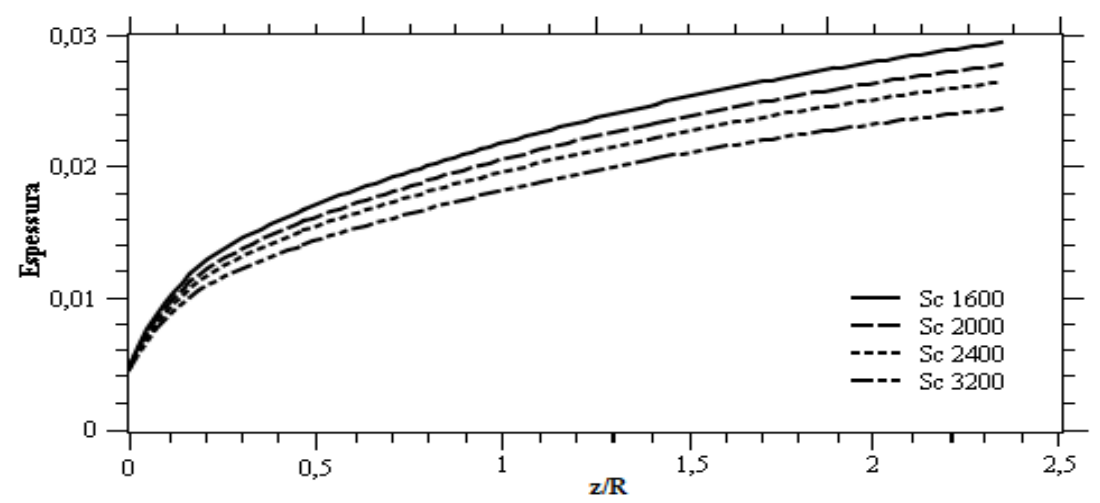

Na Figura 5, pode-se observar que quanto maior o número de Reynolds, menor a espessura da camada, tal efeito pode ser explicado pelo aumento da velocidade do sistema, ou seja, quando se aumenta o número de Reynolds a velocidade tende a aumentar e há menos acúmulo de partículas na parede permeável, este menor acúmulo se dá pelo arraste das partículas ao longo do sistema, não permitindo, assim, que o mesmo se deposite na parede permeável.

Figura 5 - Espessura da camada de polarização em função da coordenada axial para diferentes números de Reynolds

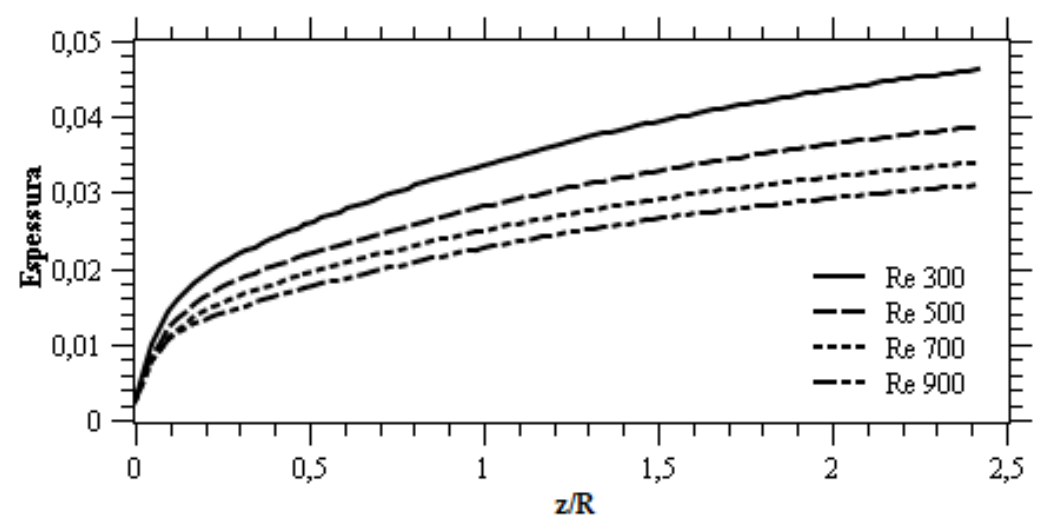

\section{CONCLUSÕES}




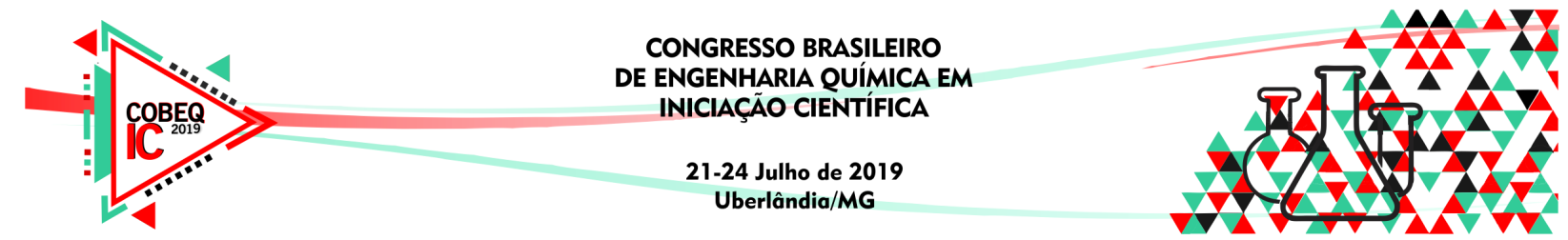

Nesse artigo, apresentou-se uma investigação de um modelo matemático utilizando-se uma malha computacional estruturada e não-uniforme para o estudo da transferência de massa associado ao processo de filtração tangencial. Com esse estudo, observou-se, com um número menor de iterações do que a malha uniforme, a região do escoamento onde a concentração de partículas é mais densa. Com essa modelagem, verificou-se que os efeitos de parâmetros físicos, número de Schmidt e Reynolds (Figuras 4 e 5), nos perfis de concentração, em região próxima à superfície permeável, possuem comportamento qualitativamente compatível com a tendência geral da literatura. Além disso, observou-se, também, que os resultados numéricos comparados com resultados analítico-numérico da literatura possuem um erro relativo médio de 5,4\%, o qual poderá diminuir com a implementação de outro esquema convectivo e/ou com a inclusão da parcela radial convectiva, $\partial c / \partial r$, na Equação (1). Portanto, tem-se que o modelo numérico proposto se mostrou razoavelmente adequado para representar o processo estudado.

\section{REFERÊNCIAS}

RIPPERGER, S.; ALTMANN, J. Crossflow microfiltration: state of the art. Separation and Purification Technology, v. 26, n. 1, p. 19-31, 2002.

ZEMAN, L. J.; ZYDNEY, A. L. Microfiltration and ultrafiltration: principles and applications. New York: Marcel Dekker, 1996.

LEE, Y.; CLARK, M. M. A numerical model of steady-state permeate flux during cross-flow ultrafiltration. Desalination,v. 109, n. 3, p. 241-251, 1997.

MIGNARD, D.; GLASS, D. H. Fouling during the cross-flow ultrafiltration of protein: a mass-transfer model. Journal of Membrane Science, v. 186, n. 1, p. 133-143, 2001.

DAMAK, K. et al. Concentration polarisation in tubular membranes: a numerical approach. Desalination, v. 171, n. 2, p. 139-153, 2004.

VENEZUELA, A. L.; PÉREZ-GUERRERO, J. S.; FONTES, S. R. Hybrid modeling of convective laminar flow in a permeable tube associated with the cross-flow process. Communications in Nonlinear Science and Numerical Simulation, v. 14, n. 3, p. 795-810, 2009.

SILVA, J. M.; FERREIRA, V. G.; FONTES, S. R. An evaluation of three upwinding approximations for numerical modeling the flow in tubular membrane of Newtonian and nonNewtonian fluids. Applied Mathematics and Computation, v. 217, n. 20, p. 7955-7965, 2011.

GERALDES, V.; SEMIÃO, V.; PINHO, M. N. Flow and mass transfer modelling of nanofiltration. Journal Membrane Science, v. 191, n. 1-2, p. 109-128, 2001.

FRANCO, N. B. Cálculo numérico. São Paulo: Pearson, 2007.

FERZIGER, J. H., PERIC, M. Computational methods for fluid dynamics. New York: Springer, 2002. 